\title{
PWM Inverter Output Filter Cost to Losses Trade Off and Optimal Design
}

\author{
Robert J. Pasterczyk \\ APC-MGE, Schneider Electric \\ Inovallée, 140 ave J. Kuntzmann \\ 38334 St Ismier CEDEX, France
}

\author{
Jean-Michel Guichon \\ Jean-Luc Schanen \\ G2Elab (UMR 5269 INPG-UJF-CNRS) \\ ENSIEG-LEG, B.P. 46 \\ 38402 St Martin d'Hères CEDEX, France
}

\author{
E. Atienza \\ Design Processing Tech. S.A. \\ Inovallée, 345 r. Lavoisier \\ 38330 Montbonnot, France
}

\begin{abstract}
This paper describes how to design the output filter of a PWM inverter used in a Uninterruptible Power Supply. Several constraints apply to this design: physical limitations of all material and components, output waveform quality, losses, cost, ... All models used in the design process are described: semiconductor, inductor and capacitor losses, cost, Total Harmonic Distortion (THD), short circuit behavior... Input parameters are control law, switching frequency, inductor design parameters (number of turns and magnetic circuit) and capacitor values. Based on these design models, optimization is carried out, and cost-losses trade-off curves are deduced. Two power levels (30kVA and 300kVA) are taken as design example, with the same models, in order to show the robustness of the method.
\end{abstract}

\section{INTRODUCTION}

\section{A. Paper Objectives}

Power electronics design is still today the property of experimented design engineers, especially in the high power range, where a poor design leads to either cost explosion or converter failure. The main problem is the multiple impacts of all design parameters on several aspects of the design. Higher switching frequency reduces the volume of passive elements, but in the same time increases losses, dropping the efficiency. Also it can lead to unacceptable loss density in the L/C components, or a too high junction temperature in the semiconductors. Considering all parameters impact together is mandatory to achieve an optimal design, since a system optimum is not reached for the optimized separately components. To account for all constraints in the same time optimization methodology has to be employed. In this paper, the output filter of a $30-300 \mathrm{kVA} 3$-ph. PWM inverter is taken as example.

\section{B. Design Constraints}

Uninterruptible Power Supply (UPS) are based on a PWM inverter part, associated with a low pass filter. The high quality of the output waveform implies a very constraint design of this LC filter. Furthermore, all losses in the power conversion are to be reduced, to increase efficiency and autonomy. On the other hand, the cost must obviously be maintained at a reasonable level. When starting to design the inverter associated with its output filter, many degrees of freedom are available: the modulation strategy, the switching frequency free or fixed, the capacitances and the inductance values. For this latter component, its custom design appears as a crucial point: choice of material, turns number, magnetic circuit size and geometry. The various constraints to be considered are

- limitations on the components (maximal losses in the semiconductors, capacitors, inductor, maximal performances of the materials, ...)

- Voltage Total Harmonic Distortion (THD) limited to $3 \%$ on R, L load, $5 \%$ on non linear load (RCD)

- Voltage drop across inductor L must not avoid to reach $230 \mathrm{~V}$ rms output voltage, for a given DC bus input voltage

- Load short circuit capability

Objective function may be either cost or efficiency. Therefore, models must be developed to compute all cost and losses. Two different inverter topologies have been studied, in order to investigate different modulation strategies (3-level or bipolar), which generate different waveforms on the inductor: inverter leg or Neutral Point Clamped (NPC) topology, illustrated in Figure 1. In order to check the validity of the models, two different power ranges have been chosen for each topology: 10kVA and 100kVA per phase.

\section{Design Methodology}

The method presented in this paper intends to start from the converter requirements only, and to propose a set of design parameters, in order to meet all these requirements, satisfying all constraints on components. Furthermore among all possible design, the best solution regarding a given criterion must be retained. To achieve this task, all equations describing the converter behaviour and all constraints are evaluated simultaneously, and all constraints checked. Thus, no hypothesis are made, contrary to a sequential design, where the designer experience is necessary to determine, for instance, the switching frequency at first, then the inductance and the resistance, satisfying all usual requirements. The global approach proposed here allows a better exploration of all possible solutions and to find the optimal one.

Having defined the design problem with an objective function (cost or efficiency) and constraints, several solving methods may be used. Two main families are available: the first one, the gradient family, necessitates the knowledge of derivative. The second one does not necessitate any specific property of the models, which can be discontinuous, and can be referred as stochastic methods. 

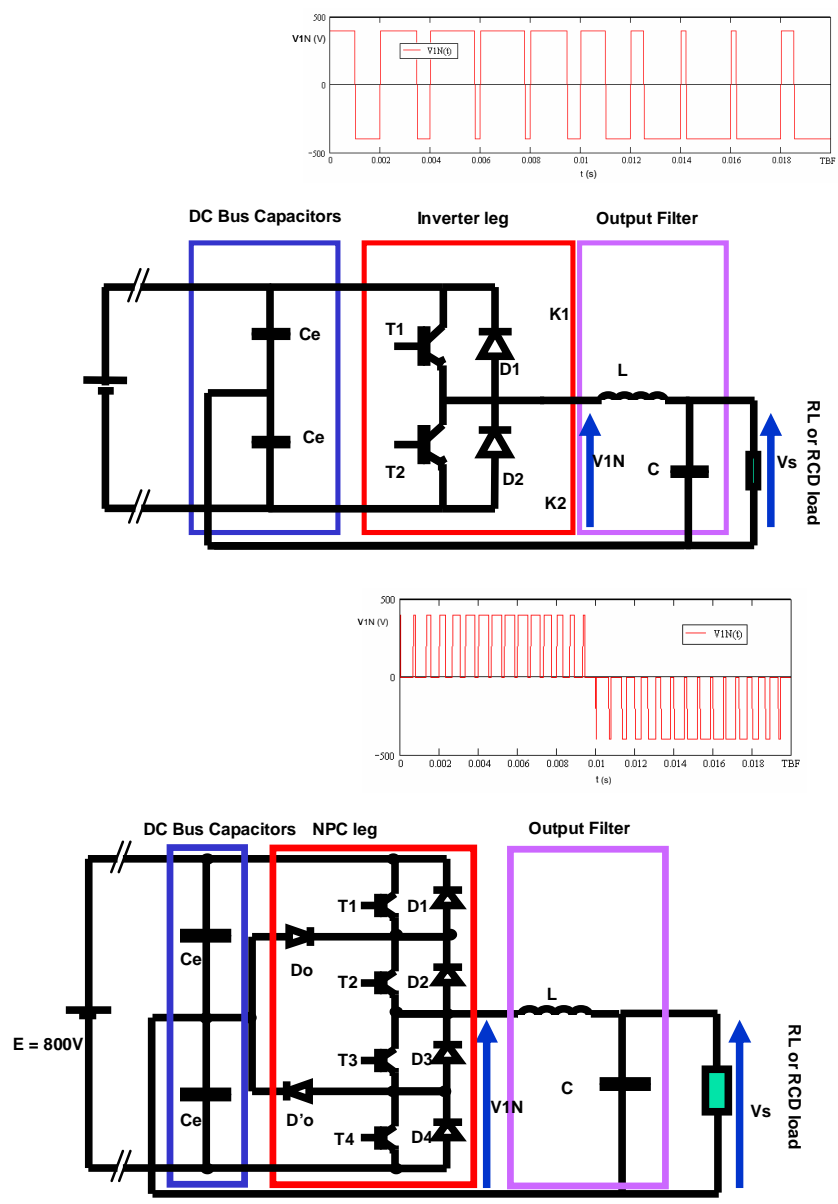

Figure 1. Two studied topologies: Dual-based inverter leg and NPC structure with the corresponding inverter output voltage waveforms
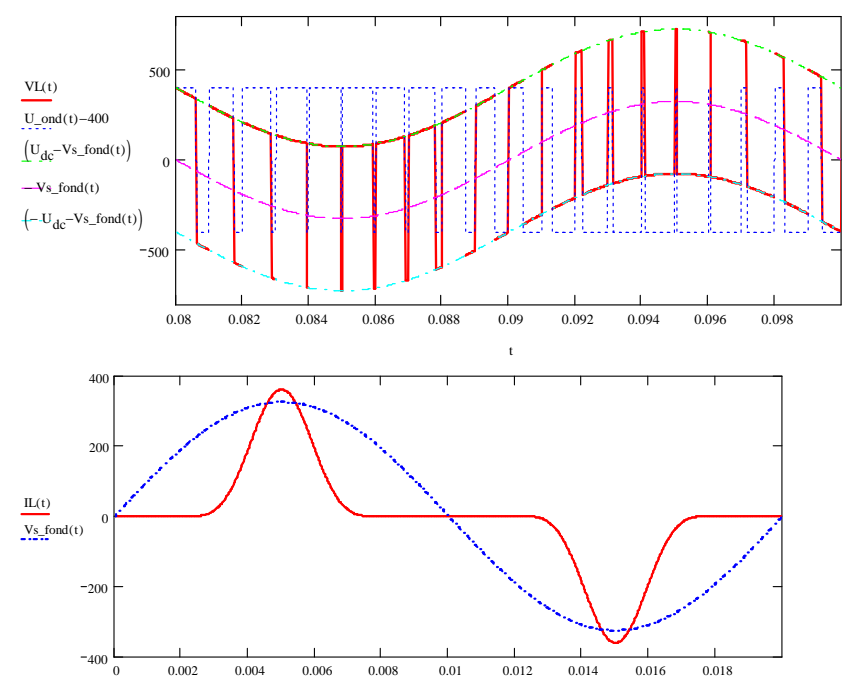

Figure 2. Example of the analytical waveforms description Voltage drop on the inductor and nonlinear RCD load current
For power electronics designers, many usual models use discrete parameters, and thus, the most popular solution is to use stochastic algorithms, very often genetic algorithms [1-4]. However, for constrained problems, with large space of solution, the gradient family is well known to be more efficient [5]. This is the general case of power electronics converters. Therefore, we chose to adapt the models to this method. It imposes to provide derivable models for all functions used in the modeling (constraints and objective function), and also to take care to the validity domain of all proposed functions, since the space of solution is wide, and all models must give physical results for any set of parameters. Physical component models, detailed in section II, are supplied by database from manufacturers regarding available component ranges and customized materials cost. From other hand all models are working under real waveforms, refer for example to Fig. 2, conditions and constraints issued from analytical description of the converter operation and its specification. Full flow-chart of the optimization process is presented in Fig. 3

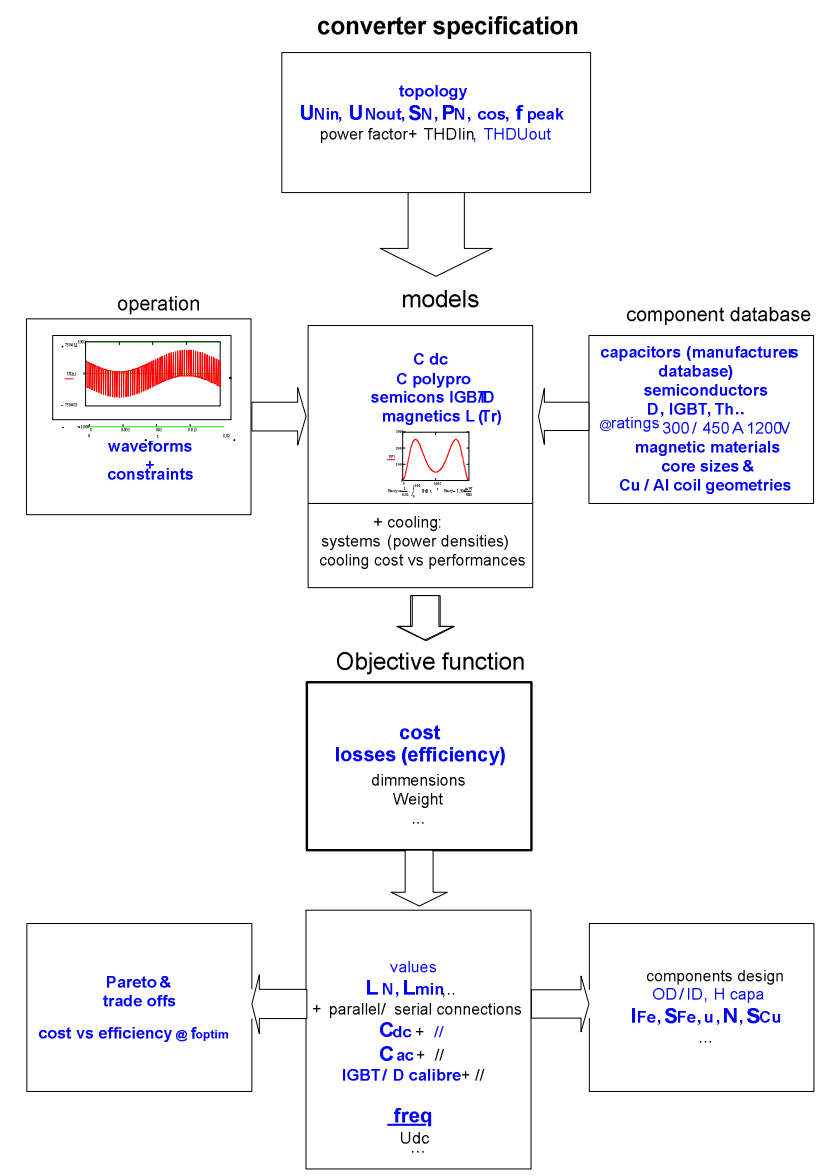

Figure 3. Methodology of the converter and its components multi-objective optimisation 


\section{COMPONENT MODELS}

Taking into account complexity all developed analytical models of the components the details on each model cannot be fully presented. We have selected the most representative models to provide a good understanding of the modeling principles (data interpolation, complex waveform simplification, temporal and frequency domain, etc..). Since the UPS standard apply generally to three types of loads RL, RC and RCD load (being representative for majority of the non linear loads) all models have been developed to account for these different cases. Transients, like inrush current of the no-load transformers, are not considered at this stage of converter conception, but of course, contribute through constraints definition.

\section{A. Capacitor model}

For the capacitor the cost and losses have to be modeled. Two technologies have been considered: electrolytic capacitors, applied on the DC bus, and polypropylene film ones on the AC side. The main useful parameters for optimization are the capacitor value and the Equivalent Series Resistance (ESR). Two different approaches have been tested to obtain these values, a complete analytical model, based on the geometry of a capacitor and its layer physical structure (Fig. 4), and manufacturer data interpolation (Fig.5). The analytical model allows a correct prediction of $\mathrm{C}$ and ESR (including skin effect and correction factor for proximity one). It is based on concentric cylinder approximation. High frequency effects in the metallization are considered using the following formula [6]:

$$
\frac{\operatorname{Rac}(n, w)}{R d c(n)}=k_{c} \cdot \frac{b(n)}{\delta(w)} \cdot \frac{\sinh \left(\frac{2 b(n)}{\delta(w)}\right)+\sinh \left(\frac{2 b(n)}{\delta(w)}\right)}{\cosh \left(\frac{2 b(n)}{\delta(w)}\right)-\cosh \left(\frac{2 b(n)}{\delta(w)}\right)}
$$

Material properties (dielectric, losses) are also accounted and included in the model. Fig. 4 shows that this analytical modeling works pretty well. Unfortunately, it implies a large number of summations across all component layers, what leads to increased computation times. Therefore, a second method, based on discrete interpolation has been preferred.

Manufacturer data have been interpolated to obtain the cost as a function of capacitance and voltage rated values. Voltage ranges appear like a parameter, as industrially only discrete values are available, for instance $2 \times 400 \mathrm{~V}$ on DC bus and $230 \mathrm{~V}$ at the output side. Similar approach has been retained for max rms current, as a function of capacitance, and the equivalent series resistance ESR.

Simple analytical functions are thus available to compute ESR, Irms_max and cost as a function of capacitance C.

Thermal model is not implemented, but thermal limitation is taken into account with a maximum fundamental and ripple HF rms current, refer to Fig. 5.
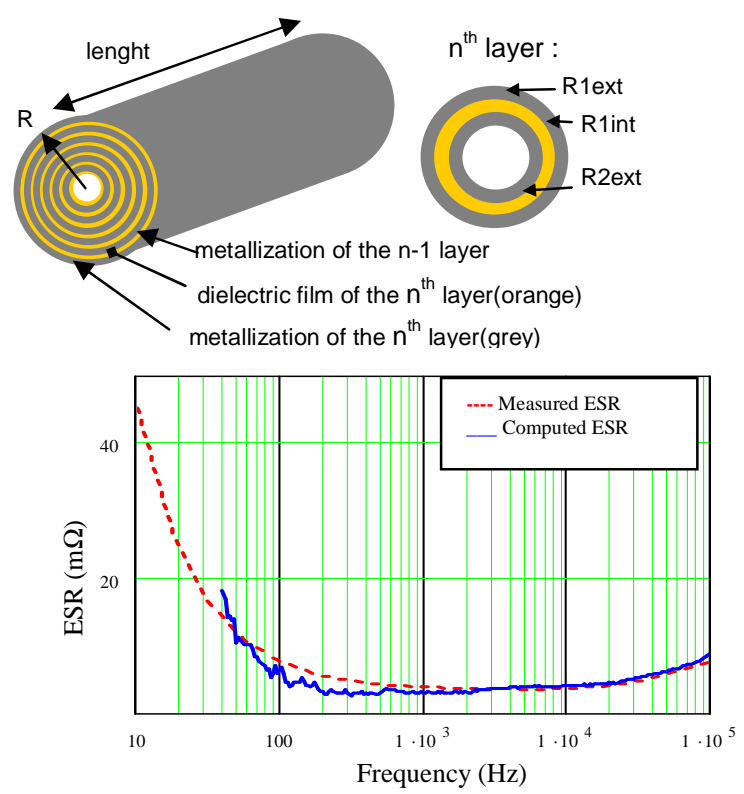

Figure 4. Geometrical modeling and full equivalent circuit of the capacitor with the validation of the ESR value

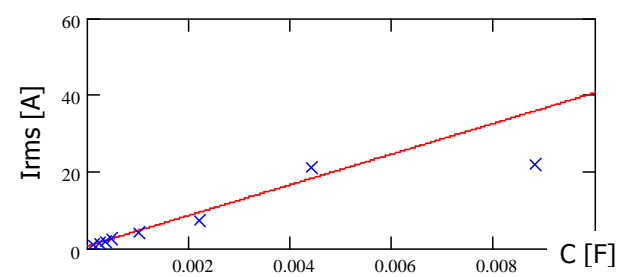

Figure 5. Example of the max rms current vs $\mathrm{C}$ rated value with datasheet and interpolation.

\section{B. Inductor model}

Contrary to capacitors, an inductor must be completely evaluated, and cannot be found as a single component in a datasheet. The description of an inductor, taken for the optimization purposes, is generic with turns number, conductor section, core section and its magnetic length, including database of nonlinear magnetic materials, as illustrates Fig. 6. Among all phenomena which must be taken into account in the design, losses and effects of the saturation are key points in such structures. This is all the more difficult since inductors are designed handling HF switching and LF fundamental voltage and current components. Manufacturer datasheets [7] are considered to determine the permeability variation, as a function of both DC or low frequency magnetization and high frequency induction variation near the current zero crossing, refer to Fig. 6. However, new formulations are to be proposed for these curves, in order to still be valid outside the material usual requirements, in order to avoid any computation and convergence errors during the optimization process. 
The physical model is used to determine the consecutive inductance variation, shown in Fig. 7, during a fundamental period in the range of $45-65 \mathrm{~Hz}$, regarding material properties and waveforms on the inductor. This variation is a key point in the design process, since it determines system current ripple, and therefore short circuit behavior, capacitor and semiconductor Safe Operating Area and losses, influencing then their ageing up to possible failure.

The core and copper volume of the inductor are determined during the design, and thus, cost can be obtained, by simply summing copper and magnetic material costs. For the cost optimal solution synthesis only the Bill of Material (BoM) is taken into account regarding this full custom component. Knowing that for high-power systems magnetic components ( $\mathrm{L}$ and $\mathrm{Tr}$ ) contribute the most to the global cost of the system.

Second particular attention was given to finely determine power losses in the magnetics, both inductors and semiconductors being the main responsible for total system efficiency. The Loss Surface behavioural model [8], based on measurements on samples of considered magnetic raw materials was developed for the converter optimal synthesis purposes. It permits accurately calculate total core losses as well as verify the H-B trajectories, shown in Fig. 8, with respect to the saturation level of used material.
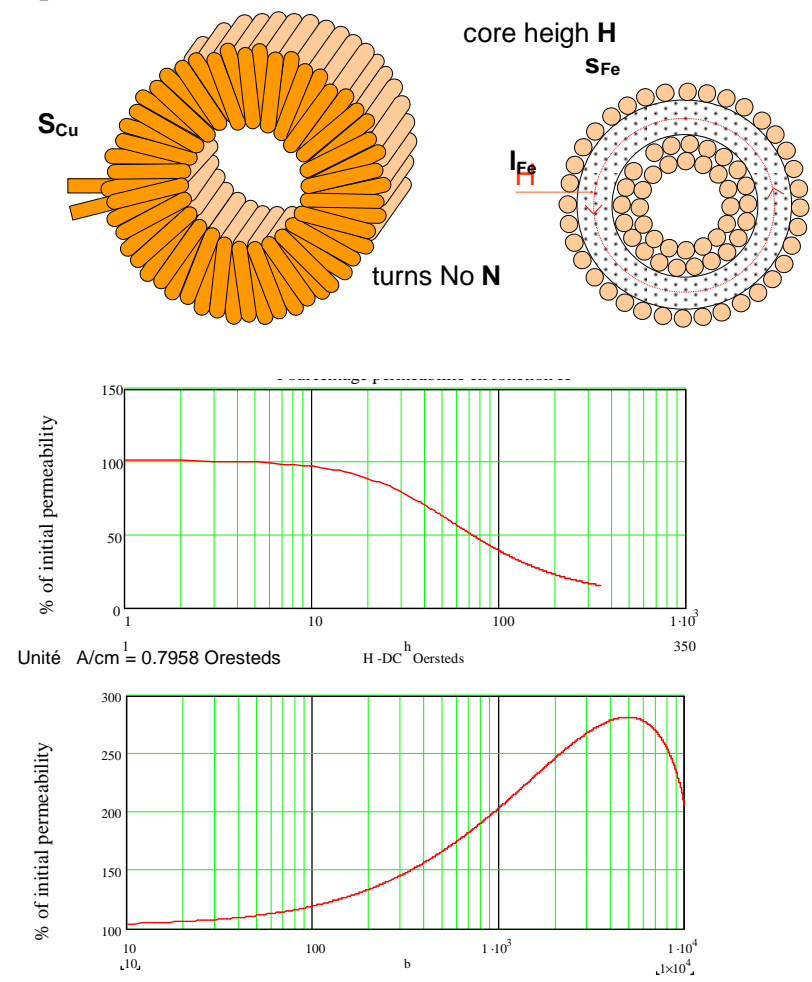

Figure 6. Geometrical and physical parameters to define an inductor Permeability variation as a function of the LF magnetization (H-DC) and HF induction (b) level near the zero current crossing

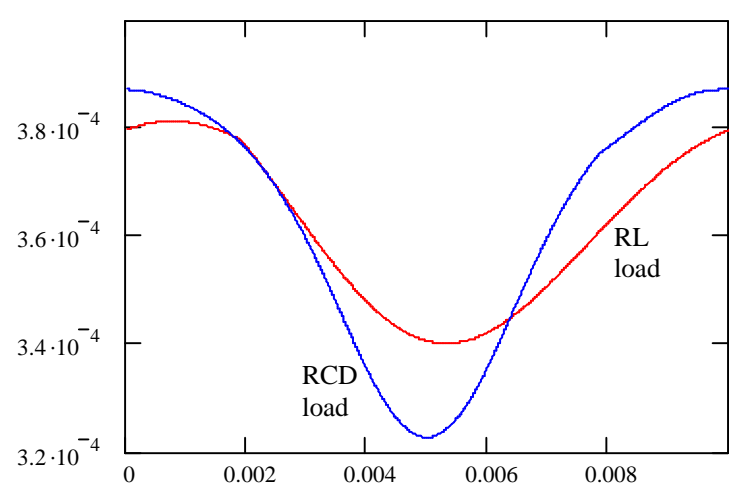

Fig. 7. Computed inductance value $[\mathrm{H}]$ for two different type of load (RL and $\mathrm{RCD})$ resulting from permeability variation, half a period shown

Losses are determinant to chose and design necessary cooling system with the additional cost coming with, which has to be evaluated and taken into account. Then the inductor model was completed by thermal aspect considering heat transfer based on toroidal inductance realization [9]. It permits to consider unidirectional radial heat flux from the center of the core and take into account at least 2-dimmensional phenomena with necessary simplification of the resulting analytical description of the model.

Construction of the thermal model with considered heat sources and thermal resistance network to its final simplification is presented in Fig. 9. Both contact and conduction resistances are taken into account, different for every layer or inductor surface knowing corresponding air flow value and direction. Heat conduction and forced convection are calculated, radiation effect is not taken into account as secondary under forced cooling conditions.

Typical values, depending on technology usually used, have been considered for thermal resistances. Even if the accuracy of such parameters is not complete, it allows avoiding the choice of inductors with an excess of loss density during the optimization. Obviously, the set of parameters depends on the envisaged realization technique, but in this predesign step, typical parameters are sufficient.

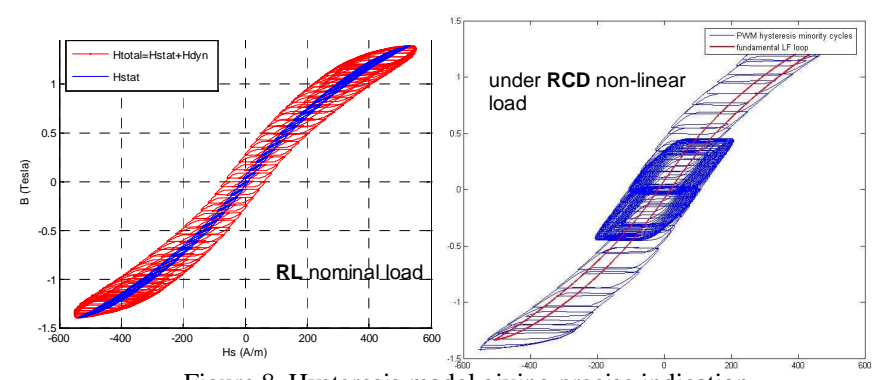

Figure 8. Hysteresis model giving precise indication of the inductor operation and core losses 

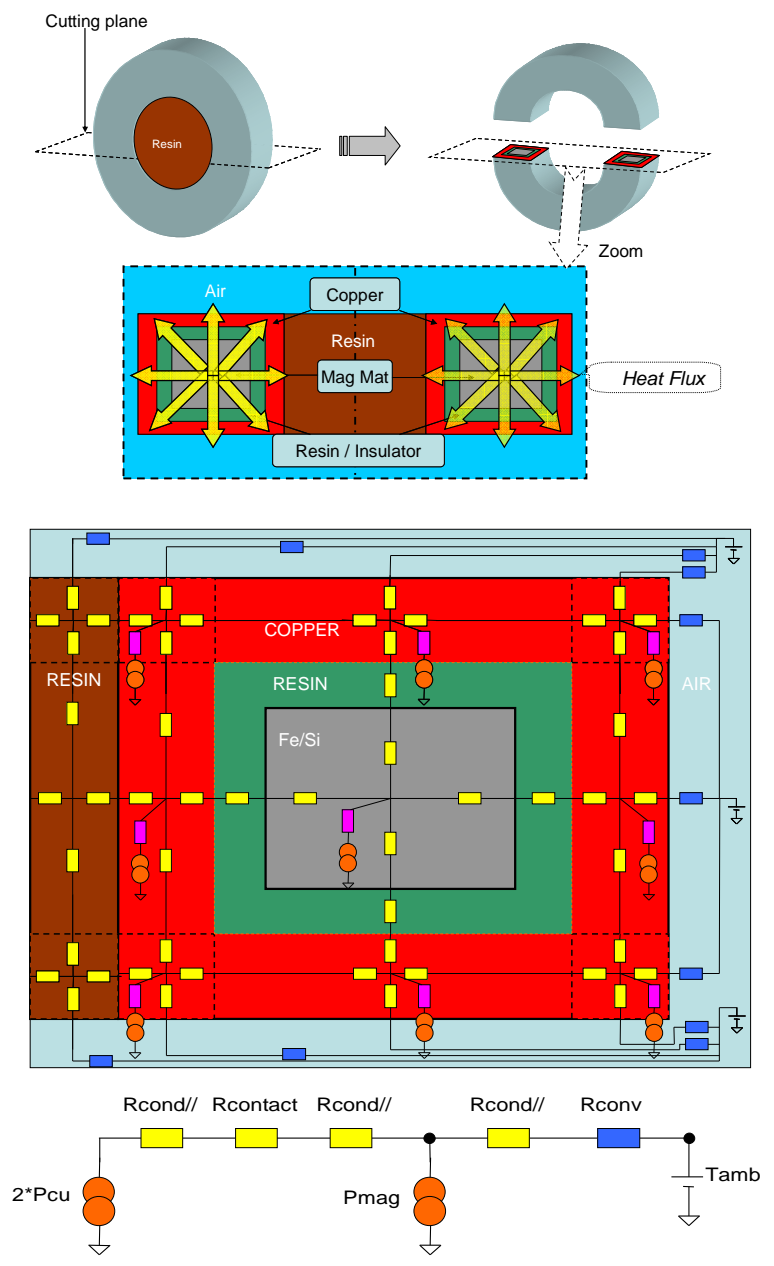

Figure 9. Thermal model based on toroidal application with corresponding thermal sources and resistances Resulting model simplification shown at the bottom

\section{Semiconductor model}

The goal of this model is to compute conduction and commutation losses. It uses well known switching energies and static voltage drop curve Vce(Ic). All data have been obtained from experimental determination on actual devices and verified with manufacturer datasheets. To be noted that during the optimization process, the space of solution can lead to a very large variation of switched current, even more than several times the nominal current: the model must still be valid at such values, even if the solution will obviously be rejected. Therefore, formula with large domain validity has been proposed to fit the experimental/datasheet results (see an example in Fig. 10).

$$
\operatorname{Eoff}(I)=a \cdot \arctan (b \cdot I) \cdot \arctan (c \cdot I)+d \cdot I
$$

Based on the knowledge of conduction and commutation losses, the conventional losses model would be to sum all switching energies of the PWM waveform, for IGBT and Diode, and adding conduction losses. However, this way of modeling lead to non continuous expressions, since the number of commutation is a discrete number.

The proposed model, illustrated in Fig. 11 first replaces the components by an equivalent leg. Within this leg, 3 commutations occur: an IGBT turn on, under the minimum current envelope, an IGBT turn off, with maximum current envelope, and a diode turn off, with minimum current. The minimum and maximum current envelopes can be expressed as continuous functions, and therefore, the leg switching losses becomes also a continuous function of the time, and the total losses can be computed using a simple integral.

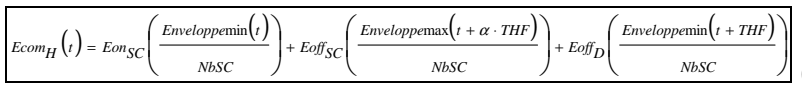

The comparison between the conventional model and this continuous model exhibit less than $2 \%$ error, even for very low switching frequencies.

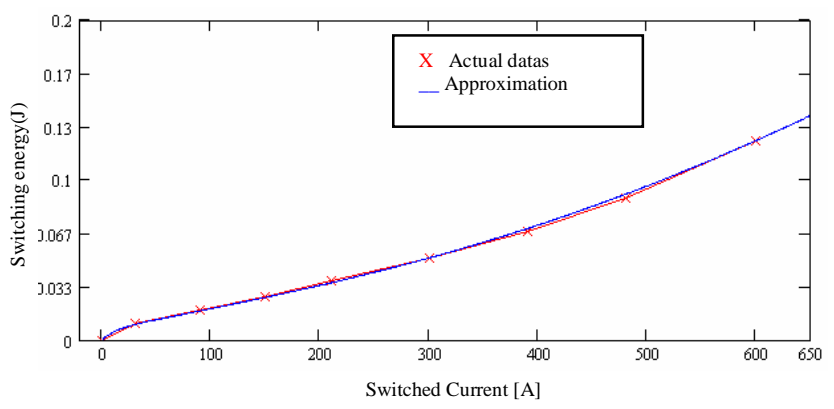

Figure 10. Example of approximation of switching energy as a function of switched current (Eon(I) for an IGBT)

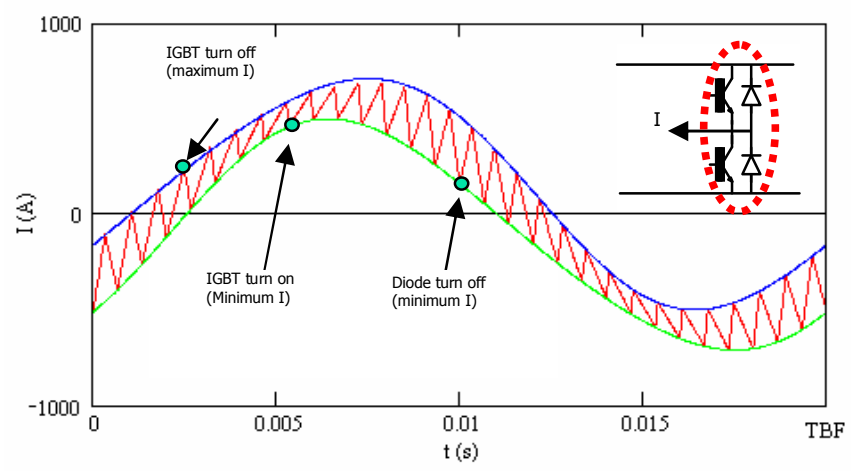

Figure 11. Equivalent model of the inverter leg to compute losses - The inductor current as a high current ripple for illustration purpose. 


\section{Peak, Average and RMS values}

For inductor design and all losses computation (inductor, capacitor, semiconductor conduction losses), there is obviously a need of computing peak, average and RMS currents. These ones depend on the structure of the converter (full bridge or NPC) and also on the proposed control. For all cases, exact continuous expressions have been developed to compute peak and average currents. For RMS current, a mathematical approximation allows to compute accurately the RMS value of PWM signal, with a large validity, even for very low switching frequencies, what is usually not the case. The principle used to obtain expression (4) is to compute first the RMS current at the time scale of High frequency period, and then to do the computation again at the time scale of low frequency. This leads to a simple integral, which can be computed easily with numerical method.

$$
I r m s=\sqrt{\frac{1}{T B F} \cdot \int_{0}^{T B F}\left(\frac{\alpha(t)}{3} \cdot\left(\operatorname{Imax}(t+T H F \cdot \alpha(t))^{2}+\operatorname{Imax}(t+T H F \cdot \alpha(t)) \cdot \operatorname{Imin}(t)+\operatorname{Imin}(t)^{2}\right)\right) \cdot d t}
$$

This method is used to compute rms current in the inductor, filter capacitor and also input capacitors. For these latter ones, the inductor average current must first be computed, in order to obtain the capacitor current.

\section{E. Voltage ripple and THD}

Voltage ripple can be easily computed using a first harmonic approximation. For THD on R, L load, an equivalent harmonic scheme has been used. To decrease computation time, the approximation has been made that the weight of all harmonics is located at the switching frequency (Fig.12). This approximation has been validated in comparison with results obtained from a temporal simulation and FFT.

THD on RCD load is computed by modeling the RDC load with its 3 first current harmonics.

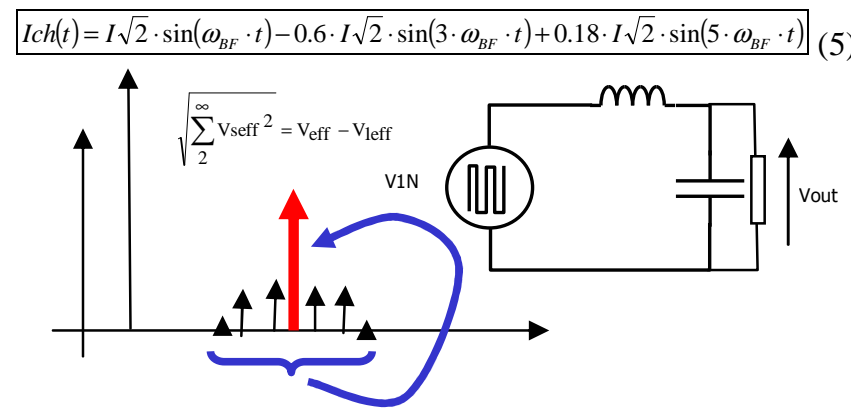

Figure 12. Harmonic scheme for THD computation and approximation

\section{OPTIMIZATION RESULTS}

The complete computation model is composed of more or less 800 equations, including all the previous models as well as all constraints, either functional or geometrical (for instance, the length of the magnetic core must not be too short, in order to be closed, even for large core area...). They have been implemented in a dedicated design software [10]. We just propose here some key trade-off curves, in order to show the interest of the results.

Fig.13 shows a typical Trade off curve for a 10kVA PWM inverter, with a low permeability material (-40, from [7]). The cost represent the L-C filter only (BoM), but all losses are included (DC capacitors, semiconductors, filter). It is clear from this figure that to reach a low cost, low volume of L-C filter is needed, thus, higher frequency is reached. On the other hand, the losses are higher... This trade off curve is very useful for design engineers.

This method can also be used to check different materials. In Fig. 14 for instance, it is clear that material -2 is better than material -8 , and that it is the best choice to reach very low losses. On the other hand, to reach low costs, material -33 should be preferred (and is better than -28 material).

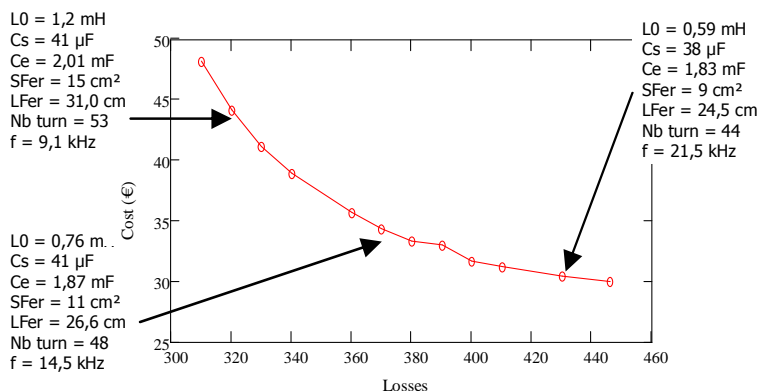

Figure 13. Trade-off result for a 10kVA PWM inverter, with -40 material [7]. Filter cost only (BoM) - all losses.

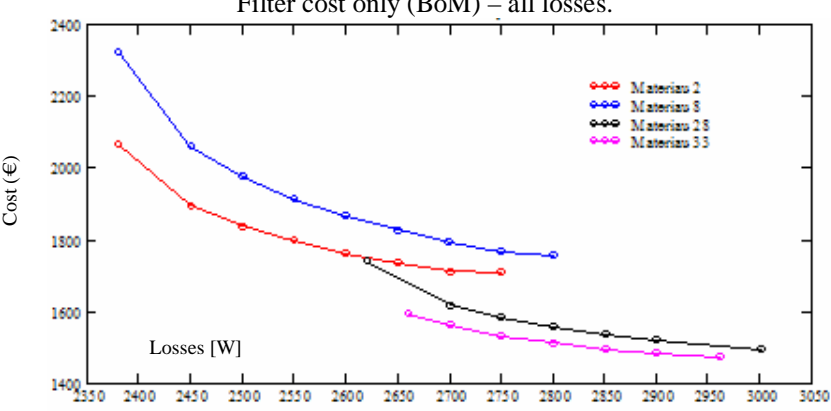

Figure 14. Comparison of different materials for a 300kVA NPC structure (filter cost only -BoM-, total losses) 
Another useful indication of the optimization results may be to compare the two structures. In Fig. 15, it is clear that from a filter point of view, the NPC structure is far more attractive, regarding losses !

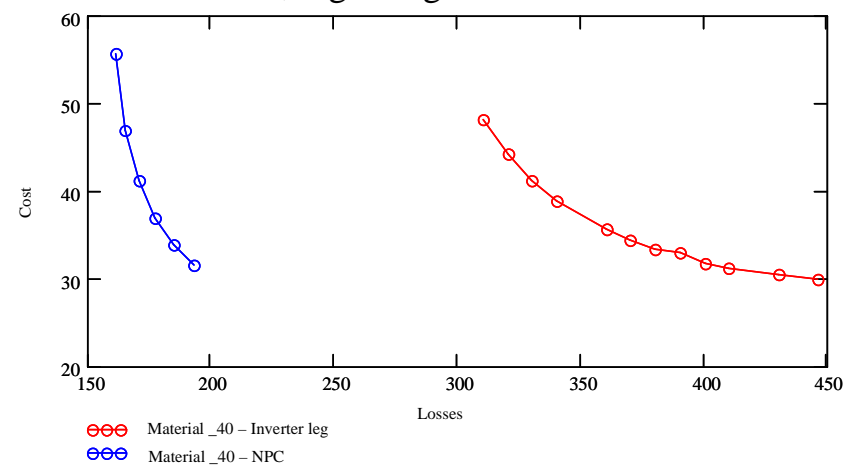

Figure 15. Comparison of the two structures for 30kVA. Material -40 (filter cost only, total losses).

\section{CONCLUSION}

In this paper, a global methodology for achieving a pre-design of a power converter has been presented. It accounts simultaneously for all parameters of the design: losses, cost, and all constraints that the converter and the components inside are faced with. Unlike previous work which use conventional models and try to find an optimization algorithm to cope with these discontinuous models, we have proposed to use gradient algorithm, more powerful to converge in large constrained spaces. Therefore, all models have to be adapted to this kind of algorithm (derivability). A specific attention has been also paid to the large validity of the models, since the algorithm is able to explore all kind of solutions, and the models must always give a physical answer.

This methodology has been applied to the design of the output filter of an Uninterruptible Power Supply, which is a very constrained element. It is very difficult is such a paper to present all the models developed for this purpose, therefore, only some key points have been underlined.
The results have been expressed as trade off curves, very useful for designers: they can be used for selecting an appropriate design, what usually can only be obtained from experimented engineers. Indeed, any parameter change can lead to a different design, and the space of solution is wide ! It is nearly impossible for a human brain to account for all the consequences of any choice during the design, except for an expert design engineer. This paper shows that this may be possible in the future, thanks to optimization procedures.

After the optimization process the results have been presented to the design engineer staff, which confirms that the current design of UPS filters is one of the points in the curve.

\section{REFERENCES}

[1] Busquets-Monge, S.; Crebier, J.-C.; Ragon, S.; Hertz, E.; Boroyevich, D.; Gurdal, Z.; Arpilliere, M.; Lindner, D.K.; "Design of a boost power factor correction converter using optimization techniques", Power Electronics, IEEE Transactions on Volume 19, Issue 6, Nov. 2004 Page(s): 1388 - 1396

[2] Busquets-monge, S.; Soremekun, G.; Hefiz, E.; Crebier, C.; Ragon, S.; Boroyevich, D.; Gurdal, Z.; Arpilliere, M.; Lindner, D.K.; " Power converter design optimization", Industry Applications Magazine, IEEE Volume 10, Issue 1, Jan-Feb 2004 Page(s):32 - 38

[3] Busquets-Monge, S.; Soremekun, G.; Hertz, E.; Crebier, C.; Ragon, S. Zhang, J.; Boroyevich, D.; Gurdal, Z.; Lindner, D.K.; Arpilliere, M.; " Design optimization of a boost power factor correction converter using genetic algorithms" Applied Power Electronics Conference and Exposition, 2002. APEC 2002 10-14 March 2002 Page(s):1177 - 1182 vol.2

[4] Helali, H.; Bergogne, D.; Slama, J.B.H.; Morel, H.; Bevilacqua, P. Allard, B.; Brevet, O.; " Power converter's optimisation and design. Discrete cost function with genetic based algorithms", Power Electronics and Applications, 2005 European Conference on 11-14 Sept. 2005 Page(s):7 pp

[5] J.Nocedal S.Wright "Numerical Optimization, Springer, ISBN 0-38798793-2

[6] P.Hammond "Applied electromagnetism.", PERGAMON, 1990.

[7] www.micrometals.com $\langle$ http://www.micrometals.com/>

[8] T. Gautreau, A. Kedous-Lebouc, T. Chevalier

Characterization and modeling of iron loss in a synchronous permanent magnet machine under no-load conditions, ICEM 2004

[9] G. Refai-Ahmed, M. M. Yovanovich, D. Cooper "Heat Transfer Modelling of Toroidal Inductors : Effects of Orientation", InterSociety Conf. on Thermal Phenomena 1998

[10] www.designprocessing.com 〈http://www.designprocessing.com/> 